\title{
Formation and evolution of different type coronal holes
}

\author{
Bilenko I.A. $\dagger$ \\ Sternberg State Astronomical Institute, Moscow, Russia, email: bilenko@sai.msu.ru
}

\begin{abstract}
Formation and evolution of coronal holes of different type are investigated. Occurrence and evolution of coronal holes in active regions are connected to the processes occurring in these active regions in particular with flares and CMEs. Coronal holes which are not connected to active regions reflect distribution of large-scale solar magnetic fields and their dynamics determined by global solar magnetic field structure changes.
\end{abstract}

Coronal holes are regions of reduced EUV and X-ray emission in the solar atmosphere. To compare coronal hole observations in different wavelengths coronal hole maps in the line $\lambda=10830 \AA$, the daily full-disk, high resolution magnetograms in the line Fe I $\lambda=8688 \AA$ obtained with the Vacuum Telescope of the National Solar Observatory Kitt Peak (Jones 1992), Yohkoh daily images, and SOHO/EUV data in ranges: $171 \AA$ (Fe IX, Fe X), $195 \AA$ (Fe XII), $284 \AA$ (Fe XV) were used. Coronal holes in different wavelengths were differed significantly. Yohkoh data showed more stability in a shape while in $10830 \AA$ it changed every day as well as area did. SOHO/EIT data have shown, that coronal holes here too are present. The coronal hole regions in He I chromospheric line coincide with unipolar magnetic field regions on the photosphere (Bohlin \& Sheeley 1978; Varsik et al. 1999). Sometimes several coronal holes in the He I line correspond to one Yohkoh coronal hole. The photospheric magnetic field structure in regions associated with He I coronal holes strongly differs from the clear regions (Belenko 2001). In those cases when whole coronal hole or its parts are observed only in the Yohkoh data and transition region $284 \AA$ line the photospheric magnetic field structure remains unchanged.

In modern models (Bohlin \& Sheeley 1978; Burlaga et al. 2001; Neugebauer \& Liewer 2003) the creation of coronal holes explained only by active region magnetic configuration, or interaction of several active region magnetic fields, or interaction of open field lines at the boundary of a polar coronal hole with closed magnetic loops outside the polar hole. In figure 1 process of occurrence of two coronal holes during 2-5 November 1998 is shown. The first coronal hole has arisen on the 3 of November on an enhanced unipolar magnetic field of positive polarity and, apparently, its occurrence directly is not connected to the NOAA 8375 active region. This coronal hole has arisen in positive unipolar longitudinal sector of coronal holes existing from the middle of 1998 up to the middle of 1999 at longitudes $180^{\circ}-270^{\circ}$. The second coronal hole has arisen on the 5 of November directly in the NOAA 8375 active region in a zone of the following negative-polarity magnetic fields. Formation of this coronal hole was probably connected to flares and CMEs observed at that time in this active region. At the subsequent rotation of the Sun this coronal hole was not observed any more. For this coronal hole a model of occurrence as a result of magnetic reconnection and formation of an area of open magnetic field configuration as a result of flare activity is suggested. As before occurrence of these coronal holes in He I

$\dagger$ Present address: Sternberg State Astronomical Institute, Universitetsky pr. 13, Moscow, Russia, 119992. 

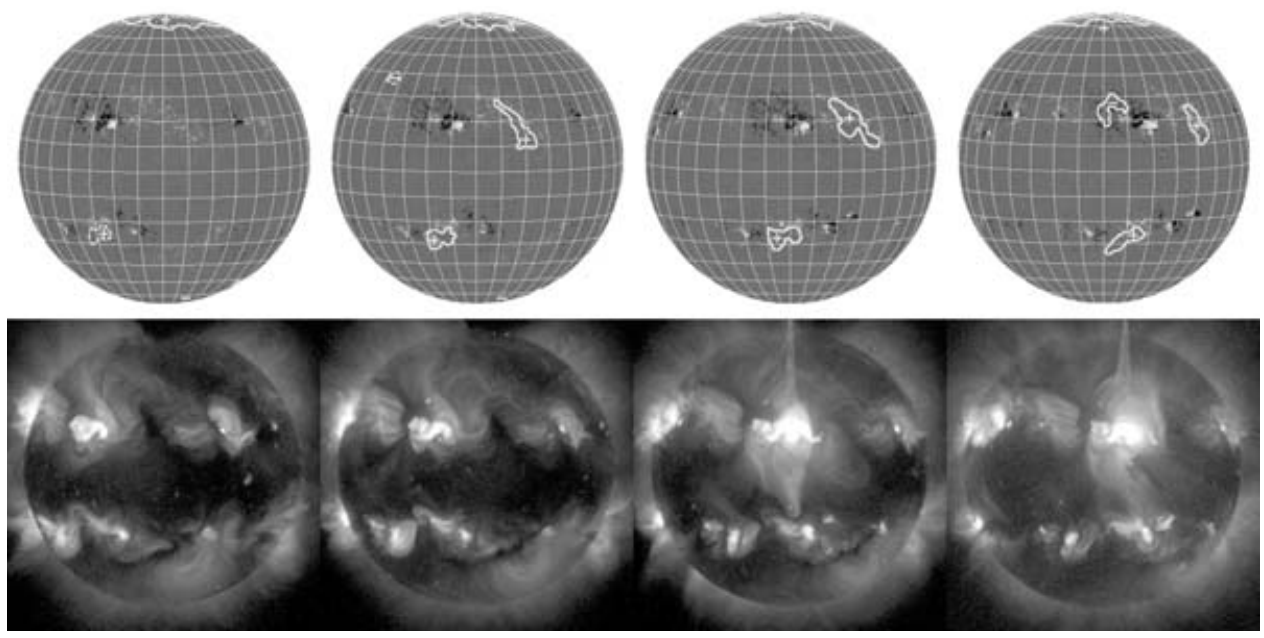

Figure 1. Magnetograms with coronal holes contours in the line $10830 \AA$ (up rows) and coronal structures from Yohkoh data (low rows) for the 2, 3, 4, 5 November 1998.

line and during their existence, together with after disappearance of the negative coronal hole, both these coronal holes were observed in the ranges $171 \AA, 195 \AA, 284 \AA$, and in coronal Yohkoh data. In the $171 \AA$ the reduced emission area even increased.

Coronal holes associated with global solar magnetic fields reflect the global magnetic field dynamics from zonal structure at the solar minimum to sectorial at maximum when an alternation of unipolar coronal hole longitudes of opposite polarity is observed (Bilenko 2002). These coronal holes first arise in the solar corona and are observed in Yohkoh data, then they penetrate to more deep layers. These holes in the lines $195 \AA, 284 \AA$ similar to Yohkoh coronal holes and in the line $171 \AA$ they similar to coronal holes in the 10830 $\AA$ line. During their evolution these coronal holes interact with active region magnetic fields. Various active regions appear and disappear during existence of separate unipolar sectors of coronal holes. But it seems that active regions are not the reason causing occurrence of these coronal holes. Dynamics of these type coronal holes determined by global solar magnetic field structure changes.

\section{Acknowledgements}

NSO/Kitt Peak data used here were produced cooperatively by NSF/NOAO, NASA/ GSFC, and NOAA/SELL. Courtesy of SOHO/EIT consortium. SOHO is a project of international cooperation between ESA and NASA. The author wish to thank the Yohkoh team for the data from their instrument.

\section{References}

Belenko, I.A. 2001 Solar Physics, 199, 23.

Bilenko, I.A. 2002 Astronomy and Astrophysics, 396, 657.

Bohlin, J.D. \& Sheeley, N.R., Jr 1978 Solar Physics, 56, 125.

Burlaga, L.F., Harvey, K.L. \& Sheeley, N.R., Jr. 2001 J.Geophys. Res. 106, 24.

Jones, H.P., Duvall, T.L., Harvey, J.W., Mahaffey, C.T., Schwitters, J.D. \& Simmons, J.E. 1992 Solar Phys. 139, 211.

Neugebauer, M. \& Liewer, P.C. 2003 J. Geophys. Res. 108, 1013.

Varsik, J.R., Wilson, P.R \& Li, Y. 1999 Solar Phys. 184, 223. 